\title{
Monitoring of the Course of Sepsis in Hematooncological Patients by Extrapituitary Prolactin Expression in Peripheral Blood Monocytes
}

\author{
P. ČEJKOVÁ ${ }^{1,2}$, V. CHROMÁ ${ }^{1}$, M. ČERNÁ ${ }^{2}$, M. MARKOVÁ ${ }^{3}$, J. MAREK $^{4}$, \\ Z. LACINOVÁ ${ }^{4}$ M. HALUZÍK ${ }^{4}$
}

${ }^{1}$ Department of Anthropology and Human Genetics, Faculty of Science, Charles University in Prague, Czech Republic, ${ }^{2}$ Department of General Biology and Genetics, Third Faculty of Medicine, Charles University in Prague, Czech Republic, ${ }^{3}$ Institute of Hematology and Blood Transfusion, Prague, Czech Republic, ${ }^{4}$ Third Department of Medicine, General University Hospital and First Medical Faculty, Charles University in Prague, Czech Republic

Received October 4, 2011

Accepted June 14, 2012

On-line August 8, 2012

\section{Summary}

Our study explored the role of extrapituitary prolactin (PRL) and toll-like receptors (TLR)2 and TLR4 in defense reaction of immune system to bacterial infection. Forty-two patients diagnosed with sepsis were recruited and blood samples were withdrawn after patients' admission to hospital, after the end of acute phase of sepsis and after the sepsis has been resolved, respectively. Seventeen patients died of sepsis; thus, only one sample collected just before death could be processed. PRL and TLR2/4 mRNA levels were measured in CD14+ blood monocytes by QPCR and PRL $-1149 \mathrm{G} / \mathrm{T}$ SNP genotyped. The TLRs mRNA expression was markedly elevated in all patients groups in comparison to healthy controls mRNA levels; the highest upregulation of monocytic TLR2 in sepsis (16.4 times, $\mathrm{P}<0.0001)$ was detected in patients who did not survive septic complications. PRL mRNA expression in monocytes from nonsurvivors tended to be lower (4.5 fold decrease, $\mathrm{P}=\mathrm{NS}$ ) compared to control levels and it was 6.2 times reduced compared to PRL mRNA expression in second blood sample from survivors $(\mathrm{P}<0.05)$. The $P R L-1149 \mathrm{G} / \mathrm{T}$ SNP had no effect on PRL mRNA response during sepsis. Our data suggest that increased prolactin mRNA expression in monocytes is associated with better outcome and improved survival rate in sepsis with no apparent effect of PRL $-1149 \mathrm{G} / \mathrm{T}$ SNP on monocytic prolactin response.

\section{Key words}

Prolactin • Toll-like receptors • mRNA • Monocytes • Sepsis

\section{Corresponding author}

Pavlina Cejkova, Department of Anthropology and Human Genetics, Faculty of Science, Charles University in Prague, Vinicna 7, 12342 Prague 2, Czech Republic. Fax: +420 221 951 618. E-mail: pavlina.cejkova@If3.cuni.cz

\section{Introduction}

Activation of innate immunity includes the production of the pattern recognition receptors (PRR) such as toll-like receptors (TLR) 2 and 4 and is considered an initial event in the immune defense response against exogenous pathogens (reviewed in Medzhitov 2001). In peripheral blood monocytes/macrophages, the activation of PRR may trigger the release of IL-1, IL-6 and TNF $\alpha$ and stimulate $\mathrm{NF} \kappa \mathrm{B}$ and JNK kinase signaling cascades followed by pro-inflammatory cytokine transcription (Salomao et al. 2008).

Sepsis, the systemic inflammatory response to severe infection frequently accompanied by multiple organ failure (MOF), is responsible for significant morbidity and mortality rate in critically ill patients. In addition to immune response, sepsis elicits a very reproducible pattern of pituitary hormone secretion, with plasma adrenocorticotropic hormone (ACTH) and prolactin (PRL) increasing within a few minutes following the insult (Sternberg 2006). The subsequent rapid activation of adrenal steroid release is a key event in the organism's first line of defense during severe illness. TLR2 and TLR4 
receptors expressed in cells of human adrenal cortex (Bornstein et al. 2004) and TLR ability to influence glucocorticoid production during sepsis propose direct effect of adrenal glands on host defense (Bornstein et al. 2006). The picture of bidirectional crosstalk between neuroendocrine and immune systems is completed by the fact that pituitary cells express PRR and can directly recognize and respond to fungal pathogen-associated molecular patterns (PAMPs). This stimulates pituitary cell TLR4 and CD14 gene expression, increases IL-6 production and PRL secretion (Breuel et al. 2004).

Contraregulatory role of prolactin against activation of adrenocortical system has been recognized for a long time. PRL may act directly on adrenal gland to drive corticosterone secretion (Glasow et al. 1996) and, PRL receptor expression in cells of adrenal cortex in several species has been discovered (Glasow et al. 1998, Glasow et al. 1996). Relative PRL insufficiency was documented in more than $50 \%$ cases of neonatal sepsis, indicating thus the significance of immunostimulatory role of prolactin in critical illness (Felmet et al. 2005). Prolactin is a plurifunctional hormone produced by pituitary gland and a number of extrapituitary tissues including immune cells T-lymphocytes, monocytes and macrophages. PRL is known to enhance monocyte, T- and B-lymphocyte immune responses under normal conditions and to have a beneficial effect on cell-mediated immunity (ChavezRueda et al. 2005, Matalka 2003, Dimitrov et al. 2004, Lahat et al. 1993). It also stimulates IFN- $\gamma$ and many other Th1-type cytokines, has comitogenic activity and prevents immune cells from apoptosis.

In this pilot study we tested the hypothesis that prolactin produced by peripheral blood monocytes is involved in the initiation and course of sepsis in hematooncological patients with potentially salutary effects on the septic patients' outcome. Furthermore, we explored the significance of TLR2 and TLR4 expression in circulating monocytes in the outcome of septic patients. To this end, we measured mRNA expression of PRL, TLR2 and TLR4 in circulating monocytes throughout the course of sepsis and tested the potential significance of PRL -1149 $\mathrm{G} / \mathrm{T}$ SNP in prolactin mRNA expression response.

\section{Methods}

\section{Subjects}

For the pilot study 42 hematooncological patients diagnosed with severe infectious event characterized by septicemia and/or multiple organ failure and hospitalized at the Institute of Hematology and Blood Transfusion in Prague, Czech Republic were recruited (age 47.14 \pm 2.20 years (mean \pm SEM); 29 male, 13 female). All included patients were treated with broad spectrum antimicrobial regimens (both anti-bacterial and anti-fungal), following the internal guidelines for antimicrobial treatment, modified according to culture results. In 25 patients, collection of their blood samples was performed three times: The first sample (A) was gained after patients' admission to the hospital; the second sample (B) after the end of acute phase, characterized by temperature normalization below $38{ }^{\circ} \mathrm{C}$, CRP decrease below $100 \mathrm{mg} / \mathrm{l}$, circulatory stabilization with no need of pressure support and no further organ involvement progression; the third sample (C) when the episode has been completely resolved, with no infectious symptoms, usually at the time of patients discharge from the hospital. The other 17 patients participating in our study did not survive septic complications and therefore, only one sample collected prior to death could be used for the study (non-survivors, N-S).

Absolute leukocyte counts (ALC) were recorded as measured by a routine hospital laboratory complete blood count. Leukopenia was defined as ALC $<4.1 \times 10^{9} / 1$ blood. For determination of infectious etiology routine cultures were performed, together with galactomannan antigen assessment in mold infection. Four groups of patients were fixed: Gram-positive $(40.5 \%)$, Gramnegative $(21.4 \%)$, mold $(9.5 \%)$, further not specified $(28.6 \%)$.

In total, 27 patients $(77.2 \%)$ underwent transplantation therapy. Leukocyte numbers in specimens collected after transplantation were in average twice as high as in comparison to specimens prior or without transplantation $(\mathrm{P}<0.05)$. Nevertheless, since the observed increase of ALC and the transplantation therapy per se did not influence the prolactin gene expression in monocytes (data not shown), we did not further include the transplantation yes/no information into our analyses.

Control group comprised 40 healthy individuals, voluntary blood donors from the Transfusion Unit of the Faculty Hospital Kralovske Vinohrady (TU FHKV), Prague, Czech Republic (age $41.13 \pm 1.77$ years (mean \pm SEM); 28 male, 12 female). Health of the control individuals and his/her eligibility for blood donation was judged according to the current TU Standard Operating Procedure: Minimum 2 weeks after complete clinical recovery from infectious disease 
(if not indicated otherwise) required; allergic persons were not eligible for blood donation during acute phase of allergic reaction or if they suffered from intermediate or severe not stabilized allergic disorder. One blood taking from each healthy subject together with his/her past history was conditioned by subjects acquainted approval. In this control group no clinical tests were performed, only DNA typing and mRNA analysis were carried out.

\section{DNA extraction and PRL -1149 G/T SNP genotyping}

Genomic DNA from peripheral blood samples collected into EDTA vacutainers was isolated by QiaAmp DNA Mini Kit spin columns (QIAGEN GmbH, Hilden, Germany) according to the manufacturer's instructions.

PRL extrapituitary SNP -1149 G/T genotyping was performed by PCR-RFLP method with originally designed oligonucleotides as described elsewhere (Fojtikova et al. 2007). After PCR amplification of $137 \mathrm{bp}$ long fragment, XapI digestion of the amplified DNA fragment was carried out at $37{ }^{\circ} \mathrm{C}$ for 2 hours and stopped by heat inactivation at $80{ }^{\circ} \mathrm{C}$ for 20 minutes. The digested and undigested products were visualized using ethidium bromide staining after electrophoresis on a $4 \%$ agarose gel.

\section{Monocyte isolation and RNA extraction}

Peripheral blood monocytes were separated by CD14 positive immunomagnetic beads (Dynal, Invitrogen, Carlsbad, $\mathrm{Ca}$, USA) following the recommended protocol. Separated monocytes were subjected to RNA isolation by RNeasy Mini Kit (QIAGEN GmbH, Hilden, Germany) with minor modification. Total amount and purity of RNA were determined by spectrophotometry.

\section{Quantitative real-time PCR ( $Q P C R)$}

For testing PRL, TLR2 and TLR4 gene expression in monocytes, ABI 7000 SDS real-time PCR instrument and TaqMan-based gene expression Assays on Demand (Applied Biosystems, Foster City, California, USA) were used: Hs00168730_m1 (PRL), Hs00152932_m1 (TLR2), Hs00152939_m1 (TLR4). As an internal control served human PGK1 (Phosphoglycerate Kinase-1) gene. The $2^{-\mathrm{dCt}}$ method was applied for relative quantification and detected mRNA values were depicted in arbitrary units (AU); in order to present acquired data uncluttered and with better resolution, values in PRL assay were multiplied by $10^{6}$ and in TLR2 and TLR4 assays by $10^{3}$.

Functionality and reliability of the PRL assay has been validated by using decidua cells as a positive control, and human HepG2 cell line that served as a negative control.

\section{Statistical analysis}

For statistical evaluation Epi Info software (Version 3.3 October 2004, Atlanta, Georgia) and GraphPadPrism software were used. To test for normality we used D'Agostino-Pearson normality test. Data are presented as median with $25 \%$ and $75 \%$ percentiles, respectively. Data involving two groups were compared using Mann-Whitney rank-sum test for unpaired values or Wilcoxon test for paired observations; data involving three or more groups were compared by Kruskal-Wallis test or Friedman test for repeated measures. Significance was accepted at $\mathrm{P}<0.05$. Allele and genotype frequencies were determined by direct counting. Statistical differences in allele or genotype distributions were analyzed by either the $\chi^{2}$ or the Fischer's exact probability test. Significance was defined using a Bonferroni corrected $\mathrm{P}$ value lower than 0.05 .

\section{Results}

Effect of -1149 G/T SNP on physiological levels PRL mRNA in healthy controls

PRL mRNA levels were compared among each genotype group in healthy controls $(\mathrm{N}=40)$. As shown in Figure 1, PRL mRNA expression in GG genotype (median 227.8 AU, 59.8-345.8) tended to be higher than in GT (median 113.2 AU, 93.4-174.5) and than in TT genotypes (median 104.8 AU, 100-285.5), respectively. However, the observed differences did not reach statistical significance $(\mathrm{P}=\mathrm{NS})$.

PRL mRNA expression in peripheral blood monocytes derived from all tested groups

Although the physiological mRNA levels of the extrapituitary PRL are in general very low (median 117.1 AU, 93.5-227; Figure 2), we observed a tendency to decreased expression in non-survivors (median 25.8 AU, 6.5-66.3; $\mathrm{P}=\mathrm{NS})$. On the contrary, PRL mRNA expression in sepsis survivors was comparable to that of healthy controls in sample A collected at the time of diagnosis (median 108.5 AU, 46.9-1011), in sample B (end of acute phase of sepsis) with a slight tendency to increase (median $160 \mathrm{AU}, 41.5-439 ; \mathrm{P}=\mathrm{NS}$ ), as well as in 
sample C (after resolution of sepsis) (median 88.1 AU, 13.5-352.6; $\mathrm{P}=\mathrm{NS}$ ) with mild decreasing tendency. However, we could observe statistically significant difference of 6.2 magnitude in median of PRL mRNA levels between non-survived patients with the lowest PRL mRNA production and median of the highest PRL mRNA expression in sample $B$ of the survived patients $(\mathrm{P}<0.05$, Figure 2).

\section{Effect of PRL $-1149 \mathrm{G} / \mathrm{T}$ SNP on physiological PRL mRNA levels}

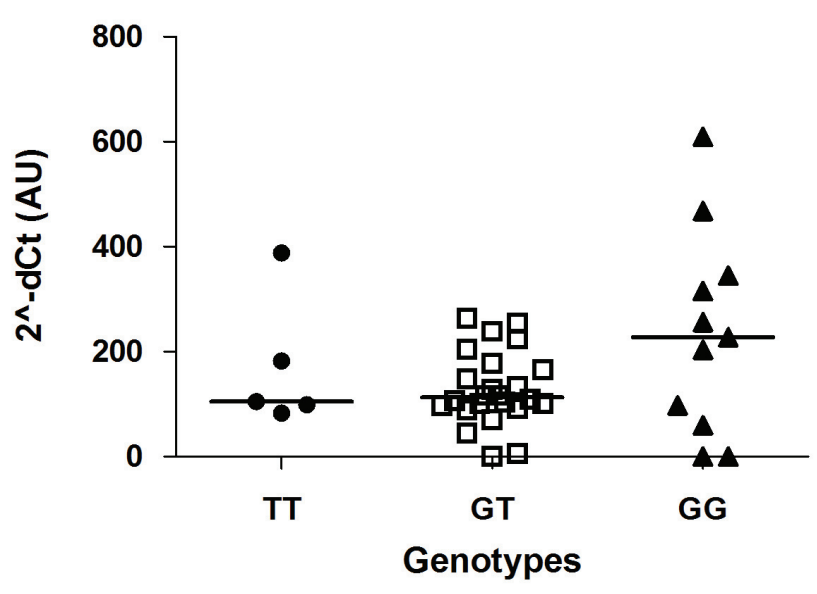

Fig. 1. Effect of $P R L-1149 \mathrm{G} / \mathrm{T}$ SNP on physiological mRNA expression in peripheral blood monocytes. The horizontal bar represents the group median. The level of mRNA expression ( $\mathrm{Y}$ axis) is shown in arbitrary units (multiplied by $10^{6}$ ).

\section{TLR2 mRNA expression}

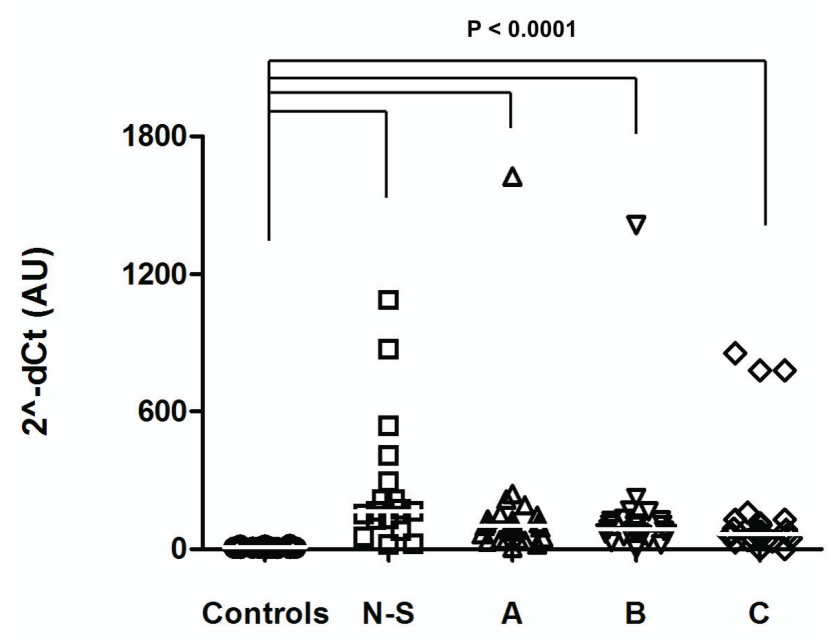

Fig. 3. Comparison of TLR2 mRNA levels of all studied groups. The horizontal bar represents the group median. Controls healthy control subjects; N-S non-survived patients; A, B, C consecutive blood drawings in patients-survivors. AU $\sim$ Arbitrary Units multiplied by $10^{3}$.

\section{PRL mRNA expression}

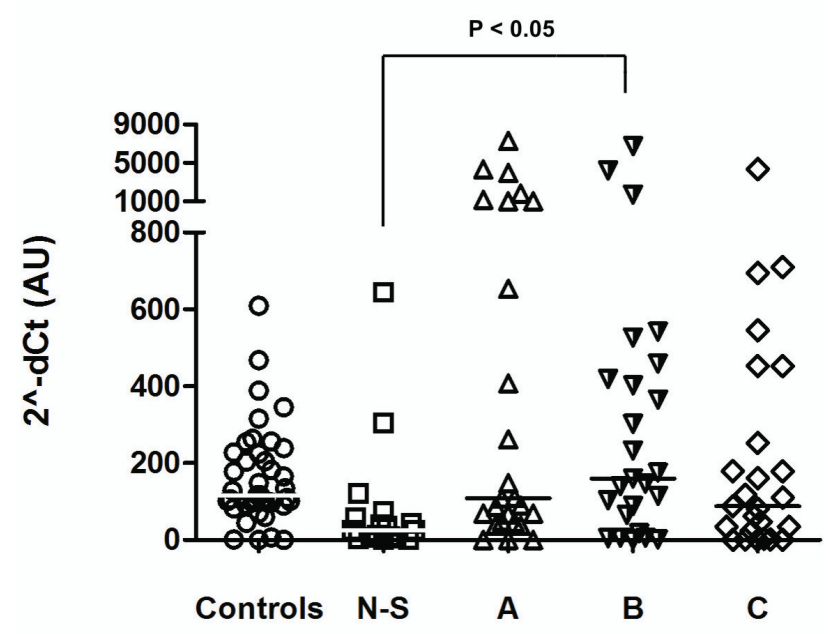

Fig. 2. Comparison of PRL mRNA levels of all studied groups. The horizontal bar represents the group median. Controls healthy control subjects; N-S $\sim$ non-survived patients; A, B, C consecutive blood drawings in patients-survivors. AU Arbitrary Units multiplied by $10^{6}$.

\section{TLR4 mRNA expression}

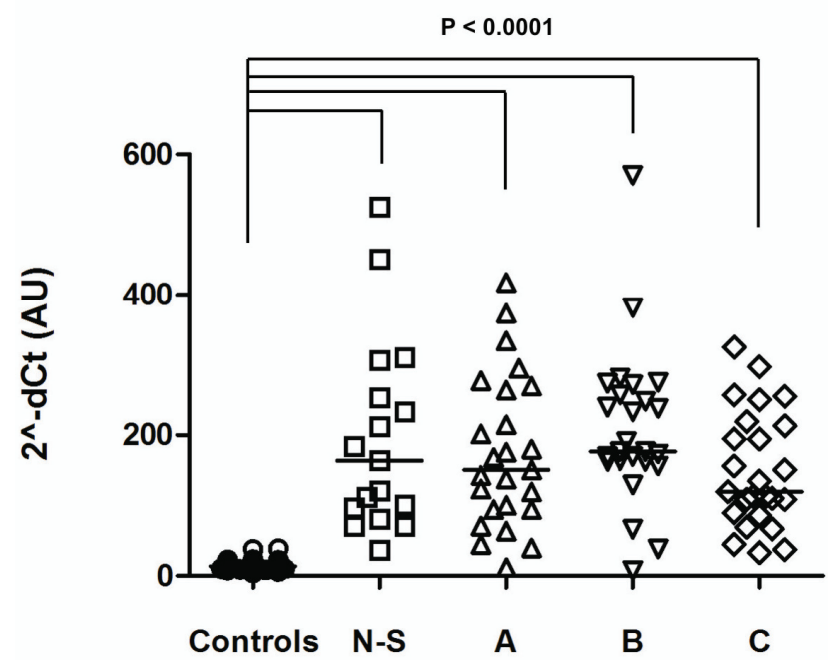

Fig. 4. Comparison of TLR4 mRNA levels of all studied groups. The horizontal bar represents the group median. Controls healthy control subjects; N-S $\sim$ non-survived patients; A, B, C consecutive blood drawings in patients-survivors. AU $\sim$ Arbitrary Units multiplied by $10^{3}$. 
TLR2 mRNA expression in peripheral blood monocytes derived from all tested groups

Both TLR2 and TLR4 mRNA expression in all studied patient groups showed steep increase compared to mRNA levels in controls (Figure 3 and 4). Interestingly, median TLR2 mRNA levels in survivors was markedly higher in comparison to healthy individuals but not as much as in non-survivors: The highest TLR2 mRNA expression was detected in non-survivors (median 166.1 AU, 104.8-352.1; 16.4 fold increase compared to control subjects, $\mathrm{P}<0.0001$ ), followed by stepwise decrease from sample A of survived patients (median 100.8 AU, 42.1138; 10 fold increase in comparison to controls, $\mathrm{P}<0.0001$ ) through sample $\mathrm{B}$ (median 86.6 AU, 63.4124.6; 8.5 fold increase when compared to controls, $\mathrm{P}<0.0001$ ) down to sample $\mathrm{C}$ (median 65.6 AU, 40.7$120.1 ; 6.5$ fold increase in comparison to control group, $\mathrm{P}<0.0001$, Figure 3).

TLR4 mRNA expression in peripheral blood monocytes derived from all tested groups

Except for samples derived from non-survived patients, the median TLR4 mRNA expression was in general higher in all studied groups than that of TLR2 (Figure 4). Healthy control subjects (median 13.9 AU, 10.7-19.8) showed 11.75 times lower mRNA expression than detected in non-survived patients (median 163.8, 88.4-279.6; $\mathrm{P}<0.0001)$. After an initial increase in sample $A$ in patients who survived septic event (median 150.7 AU, 94.1-267; 10.8 fold higher compared to control subjects, $\mathrm{P}<0.0001)$ we observed further increase in sample B (median 176.8 AU, 162.7-264.4; 12.7 fold higher compared to control subjects, $\mathrm{P}<0.0001$ ) followed by final decrease below the A sample level in sample $\mathrm{C}$ (median 119.9 AU, 87.8-216.2; 8.6 fold higher compared to control individuals, $\mathrm{P}<0.0001)$.

Pathogen type and its effect on TLR2 and TLR4 mRNA expression

Based on the pathogen type detected in patients' hemoculture we split patients into four groups: $\mathrm{G}+$ bacteria, G- bacteria, mold, not specified. Nevertheless, we were not able to find an association between any type of pathogen and level of TLR2 and TLR4 mRNA (data not shown).

\section{Discussion}

Our study was designed to evaluate the role of extrapituitary prolactin produced by peripheral blood monocytes of hematooncological patients with sepsis and to determine the impact of innate immune mechanisms represented mainly by action of Toll-like receptors on the immunological processes accompanying sepsis. Furthermore, we tested the potential significance of PRL -1149 G/T SNP on prolactin mRNA expression response to sepsis.

Previously published study carried out on Jurkat cells demonstrated that the $-1149 \mathrm{G}$ allele has changed consensual sequence and significantly increased promoter activity compared to the $-1149 \mathrm{~T}$ allele (Stevens et al. 2001). In line with this, we expected highest PRL mRNA levels associated with $\mathrm{G}$ allele and $\mathrm{GG}$ genotype. In our study, G allele in homozygous constitution tended to increase expression of $P R L$ gene (2 fold increase in comparison to TT homozygotes) but the difference did not reach statistical significance, most probably owing to relatively small sample size. Further, we focused on potential effect of the PRL $-1149 \mathrm{G} / \mathrm{T}$ SNP on PRL mRNA expression in three consecutive blood drawings in septic patients but we did not detect any specific pattern (data not shown). Taken together, our data do not support the impact of $P R L-1149 \mathrm{G} / \mathrm{T}$ genotype on the course of prolactin production by monocytes during sepsis.

It is important to note that similarly to primary human lymphocytes and other extrapituitary sites, PRL release from peripheral blood monocytes under physiological conditions is very low, compared to its pituitary production. These findings suggest specific regulatory mechanisms and different mode of action of extrapituitary prolactin from prolactin produced by anterior pituitary and also its different activity at the systemic level. It is conceivable that extrapituitary PRL acts rather locally than systemically (in lymph nodes, spleen or organs infiltrated by immune cells etc.) and thus, small amounts of immune cell-derived PRL may achieve high concentrations in the adjacent micro-environment (Montgomery 2001).

For analysis of the PRL mRNA expression in sepsis, 25 patients who survived the condition and 17 patients who died of septic complications were compared. Based on the previous results gained on pituitary prolactin (Dostal et al. 2003) we expected augmentation of PRL expression early after development of sepsis with subsequent stepwise decrease along with diminution of septic signs. However, data gained from our survivor subgroup suggest a delay in activation of PRL expression in monocytes, at least in some of examined subjects (Figure 2). We hypothesize that the 
delay in mRNA expression observed in our study may be explained by the precedential choice of utilization of intracellular PRL (IC-PRL) protein from storage vesicles as a prompt source of prolactin. Interestingly and contrary to our hypothesis, Ben Jonathan et al. (2008) found no evidence for PRL storage granules at extrapituitary sites. These authors suggested that the absence of storage granules implies constitutive PRL release rather than the calcium-dependent exocytosis like in lactotrophs. To further dissect this controversy we performed immunohistochemic staining of PBMC using monoclonal antibody against human prolactin (AbD Serotec) and in contrast to aforementioned Ben Jonathans' data we were able to see IC-PRL localized mainly in close vicinity to nucleus and in $25 \%$ of cells also in vesicle-like depots in cytoplasm (data not shown).

In 17 patients who died of sepsis, the pattern of PRL mRNA expression was different from sepsis survivors. We observed non-significant tendency towards decrease in PRL mRNA expression compared to healthy subject levels and even 6.2 times lower expression in comparison with the highest PRL mRNA levels detected in the second blood drawing $\mathrm{B}$ from survivor subgroup $(\mathrm{P}<0.05)$. It has already been demonstrated that prolactin is important for maintaining normal immune response in several pathological states; moreover, PRL ability to improve survival of animals subjected to sepsis after hemorrhage has been documented (Zellweger et al. 1996b, Zellweger et al. 1996a). Our finding is in line with results of work by Felmet and colleagues who studied the effect of hypoprolactinemia in children with nosocomial sepsis. Felmet et al. (2005) reported that hypoprolactinemia aggravated septic condition and that PRL inhibited stress-induced apoptosis of lymphocytes, confirming the significance of PRL anti-apoptotic effect.

Since our data revealed tendency to the lowest PRL mRNA levels in monocytes from patients who died of sepsis, we found it interesting to discover any potential correlation between mortality in sepsis and $P R L-1149 \mathrm{G} / \mathrm{T}$ genotypes. Nevertheless, there was no effect of studied SNP on patients' survival (data not shown). Despite the known impact of $P R L-1149$ alleles on $P R L$ transcription in immune cells (Stevens et al. 2001) our data did not show any effect of this polymorphism on PRL mRNA levels.

The innate immune system, particularly Toll-like receptors provide the first line of defense against invading bacteria and other pathogens (for review, see Knapp 2010). Monocytes are the key cellular component of the innate immunity with high expression of Toll-like receptors in response to bacterial infection and sepsis and are of great importance in immune system defense against various pathogens (Haveman et al. 1999, Jiang et al. 2005). Here we show, similarly with previously published results (Tsujimoto et al. 2004, Armstrong et al. 2004), that both TLR2 and TLR4 mRNA monocytic expression was upregulated in all groups of patients in comparison to the basal state in healthy subjects. This finding mirrors the ongoing inflammation and the immune system response against infection. We observed higher increase in TLR2 mRNA expression in nonsurvivor compared to survivor group suggesting inappropriate response of non-survivors' immune system before death. In survivor group, gradual decrease in $T L R$ genes mRNA expression along with diminution of clinical signs of sepsis was observed. Intriguingly, even in sample $\mathrm{C}$ withdrawn after resolution of septic episode and confirmed clinical improvement, neither TLR2 nor TLR4 mRNA levels reached the basal levels comparable to those in healthy controls.

In monocytes, CD14 receptor facilitates TLR2 and TLR4 activation through transferring TLR-specific ligands (Wright et al. 1990). However, there is increasing evidence that bacterial peptides are not the only ligands that can activate TLR2 and TLR4. It has been demonstrated that TLR2 and TLR4 are involved also in trauma where Gram-negative or Gram-positive bacteria were not detected in circulation or local organ sites (Lorne et al. 2010). This fact may explain our finding of activation of TLR2 and TLR4 mRNA in all septic patients regardless of type of pathogen detected in hemoculture. Interestingly, we did not see a clear relationship between Gram-negative hemoculture and TLR4, or between Gram-positive hemoculture and TLR2 mRNA upregulation, respectively.

Collectively, our data together with previously published observations show 4.5 times lowered PRL mRNA levels in monocytes derived from non-survived septic patients, and statistically significantly 6.2 times lower expression in comparison with the highest PRL mRNA levels detected in monocytes derived from survived patients. The 1.5 fold upregulation of PRL mRNA expression detected in survived patients at the end of acute phase of sepsis when compared to healthy controls reflects an ongoing immune activation of stressed organism. These data confirm the significance of prolactin for better outcome and improved survival rate in sepsis and suggest that extrapituitary prolactin plays an important role in the activation of the innate immune 
system during bacterial infection. In our study, we were not able to show the involvement of functional $P R L$ -1149 G/T SNP and its significance for monocytic prolactin mRNA expression regulation. Our data also indicate that the expression of Toll-like receptors mRNA during sepsis is markedly increased in comparison to mRNA levels in healthy controls, which reflects general response of organism to infection.

\section{Conflict of Interest}

There is no conflict of interest.

\section{Acknowledgements}

This study was supported by the grant IGA NS 9970-4. The authors would like to thank to Katerina Janatova for her laboratory assistance.

\section{References}

ARMSTRONG L, MEDFORD ARL, HUNTER KJ, UPPINGTON KM, MILLAR AB: Differential expression of Tolllike receptor (TLR)-2 and TLR-4 on monocytes in human sepsis. Clin Exp Immunol 136: 312-319, 2004.

BEN-JONATHAN N, LAPENSEE CR, LAPENSEE EW: What can we learn from rodents about prolactin in humans? Endocr Rev 29: 1-41, 2008.

BORNSTEIN SR, SCHUMANN RR, RETTORI V, MCCANN SM, ZACHAROWSKI K: Toll-like receptor 2 and Tolllike receptor 4 expression in human adrenals. Horm Metab Res 36: 470-473, 2004.

BORNSTEIN SR, ZIEGLER CG, KRUG AW, KANCZKOWSKI W, RETTORI V, MCCANN SM, WIRTH M, ZACHAROWSKI K: The role of toll-like receptors in the immune-adrenal crosstalk. Ann N Y Acad Sci 1088: 307-318, 2006.

BREUEL KF, KOUGIAS P, RICE PJ, WEI D, DE PONTI K, WANG J, LAFFAN JJ, LI C, KALBFLEISCH J, WILLIAMS DL: Anterior pituitary cells express pattern recognition receptors for fungal glucans: implications for neuroendocrine immune involvement in response to fungal infections. Neuroimmunomodulation 11: 1-9, 2004.

CHAVEZ-RUEDA K, HERNANDEZ J, ZENTENO E, LEANOS-MIRANDA A, LEGORRETA-HAQUET MV, BLANCO-FAVELA F: Identification of prolactin as a novel immunomodulator on the expression of costimulatory molecules and cytokine secretions on T and B human lymphocytes. Clin Immunol 116: 182-191, 2005.

DIMITROV S, LANGE T, FEHM HL, BORN J: A regulatory role of prolactin, growth hormone, and corticosteroids for human T-cell production of cytokines. Brain Behav Immun 18: 368-374, 2004.

DOSTAL C, MOSZKORZOVA L, MUSILOVA L, LACINOVA Z, MAREK J, ZVAROVA J: Serum prolactin stress values in patients with systemic lupus erythematosus. Ann Rheum Dis 62: 487-488, 2003.

FELMET KA, HALL MW, CLARK RS, JAFFE R, CARCILLO JA: Prolonged lymphopenia, lymphoid depletion, and hypoprolactinemia in children with nosocomial sepsis and multiple organ failure. J Immunol 174: 3765-3772, 2005.

FOJTIKOVA M, CERNA M, CEJKOVA P, RUZICKOVA S, DOSTAL C: Extrapituitary prolactin promoter polymorphism in Czech patients with systemic lupus erythematosus and rheumatoid arthritis. Ann Rheum Dis 66: 706-707, 2007.

GLASOW A, BREIDERT M, HAIDAN A, ANDEREGG U, KELLY PA, BORNSTEIN SR: Functional aspects of the effect of prolactin (PRL) on adrenal steroidogenesis and distribution of the PRL receptor in the human adrenal gland. J Clin Endocrinol Metab 81: 3103-3111, 1996.

GLASOW A, HAIDAN A, GILLESPIE J, KELLY PA, CHROUSOS GP, BORNSTEIN SR: Differential expression of prolactin receptor (PRLR) in normal and tumorous adrenal tissues: separation of cellular endocrine compartments by laser capture microdissection (LCM). Endocr Res 24: 857-862, 1998.

HAVEMAN JW, MULLER KOBOLD AC, TERVAERT JW, VAN DEN BERG AP, TULLEKEN JE, KALLENBERG CG, THE TH: The central role of monocytes in the pathogenesis of sepsis: consequences for immunomonitoring and treatment. Neth J Med 55: 132-141, 1999. 
JIANG ZF, GEORGEL P, DU X, SHAMEL L, SOVATH S, MUDD S, HUBER M, KALIS C, KECK S, GALANOS C, FREUDENBERG M, BEUTLER B: CD14 is required for MyD88-independent LPS signaling. Nat Immunol 6: $565-570,2005$.

KNAPP S: Update on the role of Toll-like receptors during bacterial infections and sepsis. Wien Med Wochenschr 160: 107-111, 2010.

LAHAT N, MILLER A, SHTILLER R, TOUBY E: Differential effects of prolactin upon activation and differentiation of human B-lymphocytes. J Neuroimmunol 47: 35-40, 1993.

LORNE E, DUPONT H, ABRAHAM E: Toll-like receptors 2 and 4: initiators of non-septic inflammation in critical care medicine? Intensive Care Med 36: 1826-1835, 2010.

MATALKA KZ: Prolactin enhances production of interferon-gamma, interleukin-12, and interleukin-10, but not of tumor necrosis factor-alpha, in a stimulus-specific manner. Cytokine 21: 187-194, 2003.

MEDZHITOV R: Toll-like receptors and innate immunity. Nat Rev Immunol 1: 135-145, 2001.

MONTGOMERY DW: Prolactin production by immune cells. Lupus 10: 665-675, 2001.

SALOMAO R, MARTINS PS, BRUNIALTI MK, FERNANDES ML, MARTOS LS, MENDES ME, GOMES NE, RIGATO O: TLR signaling pathway in patients with sepsis. Shock 30 (Suppl 1): 73-77, 2008.

STERNBERG EM: Neural regulation of innate immunity: a coordinated nonspecific host response to pathogens. Nat Rev Immunol 6: 318-328, 2006.

STEVENS A, RAY D, ALANSARI A, HAJEER A, THOMSON W, DONN R, OLLIER WER, WORTHINGTON J, DAVIS JRE: Characterization of a prolactin gene polymorphism and its associations with systemic lupus erythematosus. Arthritis Rheum-US 44: 2358-2366, 2001.

TSUJIMOTO H, ONO S, HIRAKI S, MAJIMA T, KAWARABAYASHI N, SUGASAWA H, KINOSHITA M, HIRAIDE H, MOCHIZUKI H: Hemoperfusion with polymyxin B-immobilized fibers reduced the number of CD16(+)CD14(+) monocytes in patients with septic shock. J Endotoxin Res 10: 229-237, 2004.

WRIGHT SD, RAMOS RA, TOBIAS PS, ULEVITCH RJ, MATHISON JC: CD14, a receptor for complexes of lipopolysaccharide (LPS) and LPS binding protein. Science 249: 1431-1433, 1990.

ZELLWEGER R, WICHMANN MW, AYALA A, DEMASO CM, CHAUDRY IH: Prolactin: a novel and safe immunomodulating hormone for the treatment of immunodepression following severe hemorrhage. J Surg Res 63: 53-58, 1996a.

ZELLWEGER R, ZHU XH, WICHMANN MW, AYALA A, DEMASO CM, CHAUDRY IH: Prolactin administration following hemorrhagic shock improves macrophage cytokine release capacity and decreases mortality from subsequent sepsis. J Immunol 157: 5748-5754, 1996b. 